\title{
Acceleration of bone-defect repair by using A-W MGC loaded with BMP2 and triple point-mutant HIF1a-expressing BMSCs
}

Yuzhong Gao ${ }^{1,2}$, Chen $\mathrm{Li}^{3}$, Hao Wang ${ }^{2}$ and Guangyu Fan ${ }^{1 *}$

\begin{abstract}
Background: The goal of this study is to explore the effects of A-W MGC (apatite-wollastonite magnetic bioactive glass-ceramic) loaded with BMP2 (bone morphogenetic protein 2)- and HIF1a ${ }^{\text {mu }}$ (hypoxia-inducible factor 1 mutation)expressing BMSCs (bone marrow mesenchymal stem cells) on the bone defect repair.

Methods: (1) BMSCs were infected with viral solution containing BMP2 and HIF1a ${ }^{\text {mu }}$ with the best MOI (multiplicity of infection). The efficiency was observed via hrGFP (human renilla reniformis green fluorescent protein). (2) The cells were divided into five groups (A-E), and ALP (alkaline phosphatase) activity was measured. (3) BMP2 and HIF1a (hypoxia-inducible factor 1a) protein were measured. (4) A-W MGC was loaded with BMSCs that contain the genes and implanted into the bone defect model. The animals were sacrificed 8 and 12 weeks later. (5) The healing was measured with X-ray, histology, and biomechanics.
\end{abstract}

Results: (1) BMSCs in A-D showed high transfection efficiency. (2) ALP in A and B was higher than the others ( $p=0.041$ or 0.038$)$; $A$ was higher than $B(p=0.038)$; (3) BMP2 in A and B was higher than the others $(p=0.014)$. HIF1a in A and C was higher than the others $(p=0.020)$. (4) 8 and 12 weeks after, an $X$-ray indicated that bone defect was nearly fully repaired in $A$ and $C$. (5) 12 weeks after, the bone remodeling was complete in A and C. (6) The flexural strength in $A$ and $C$ was stronger than the others $(p=0.043)$.

Conclusion: Engineered A-W MGC with BMP2 and HIF1a ${ }^{\text {mu}}$-expressing BMSCs exhibits comparable therapeutic effects of bone-defect repair as an autologous bone graft.

Keywords: Bone defects, Bone marrow mesenchymal stem cells, Bone morphogenetic protein 2, Hypoxia-inducible factor $1 a$

\section{Background}

Treatment of large bone defects caused by trauma has been a major unsolved problem in the orthopedics field $[1,2]$. Clinically, bone grafts have remained the standard treatment to restore the integrity and biomechanical properties of the bone defects. The breakthrough in creating a new type of engineered bone grafts, which combine the osteogenic factors, osteogenic stem cells, and the artificial bone scaffold, provides a new way for the bone-defect treatment.

\footnotetext{
* Correspondence: guangyufancmd@sina.com

${ }^{1}$ Department of Orthopedics, The Affiliated First Hospital of China Medical University, No. 155, Nanjing North Street, Heping District, 110001 Shenyang, Liaoning province, China

Full list of author information is available at the end of the article
}

BMP (bone morphogenetic protein) is one of the osteogenic factors that play important roles in the ontogenesis. BMP can stimulate the differentiation of mesenchymal cells into osteogenic cells and enhance osteoblasts [3], which initiates a series of bone regeneration steps to improve bone healing. However, due to the damage of the blood vessels of the microcirculation system at the injury site, it is difficult to rebuild a vascular system in the core part of the large engineered bone graft (diameter $>5 \mathrm{~mm}$ ), resulting in poor bone growth and the failure of bone graft transplantation. Meanwhile, the transfected osteogenic cells stop growing due to insufficient nutrient supply, resulting in the difficulty of new bone formation inside the engineered bone graft. Therefore, it is critical to build an effective network of 
blood vessels within the bone graft as early as possible and form sufficient connections with the blood vessels in the host bones to provide nutritional and metabolic supply and stimulate osteogenic differentiation, thereby promoting healing and repair of bone defects.

Angiogenesis is regulated by a series of growth factors [4-6]. Because HIF1 $\alpha$ (hypoxia-inducible factor $1 \alpha$ ) plays an important role in promoting angiogenesis, it is considered the most promising gene in the clinical application [7-11]. However, HIF1 $\alpha$ can only be expressed and accumulated under hypoxic conditions. The 402 and 564 proline in the ODDD (oxygen-dependent degradation domain) in the CDS (coding sequence) cannot be hydroxylated, which causes the rapid degradation (within minutes) of the endogenous HIF1 $\alpha$ [12-15]. Lando et al. have reported [16] that the transcriptional activity of HIF1 $\alpha$ can be reduced by the function of the 803 asparagine in the CDS area, suggesting that the 402 and 564 proline and the 803 asparagine in the CDS area are important loci of HIF1 $\alpha$. In our previous research, we have created HIF $1 \alpha^{\mathrm{mu}}$ (hypoxia-inducible factor 1 mutation), which not only can be expressed under normoxic condition in vitro but also can promote angiogenesis in the bone-defect area under normoxic conditions in vivo [17]. In this current study, we constructed engineered bone graft using A-W MGC (apatite-wollastonite magnetic bioactive glass-ceramic) combined with BMSCs that are co-infected with adenovirus vectors containing HIF $1 \alpha^{\mathrm{mu}}$ and BMP2 genes. Our goal is to explore the interaction of HIF $1 \alpha^{\text {mu }}$ and BMP2 and the impact on angiogenesis and ontogenesis in bone-defect area.

\section{Materials and methods}

\section{Materials}

The reagents used in this study are shown in Table 1.

\section{Animals}

Sixty-one purebred New Zealand white rabbits, 3-4 months of age, weighing $2.0-2.8 \mathrm{~kg}$, male or female, were provided by the Experimental Animal Center of Liaoning Medical University. All experimental procedures were carried out in accordance with the experimental animal and animal welfare requirements of Liaoning Medical Experiments Animal Ethics Committee.

\section{Methods}

\section{Infection of BMSCs with recombinant adenovirus}

The New Zealand white rabbit was deeply anesthetized, and the bone marrows from the right tibial bone were harvested. BMSCs were isolated, purified, and cultured to the third generation. The infections of Adv-HIF1 $\alpha^{\mathrm{mu}}$ hrGFP and Adv-BMP2-hrGFP were carried out with the best MOI (multiplicity of infection): $\mathrm{MOI}=100(\mathrm{HIF} 1 \alpha)$ and $\mathrm{MOI}=150$ (BMP2). The infection efficiency was
Table 1 Reagents

\begin{tabular}{ll}
\hline Name & Company \\
\hline Adv-HIF1a ${ }^{\text {mu }}$ hrGFP $\left(1.6 \times 10^{8} \mathrm{pfu} / \mathrm{mL}\right)$ & First Affiliated Hospital of \\
& Liaoning Medical Tissue \\
& Engineering Laboratory \\
Adv-BMP2-hrGFP $\left(2.0 \times 10^{8} \mathrm{pfu} / \mathrm{mL}\right)$ & First Affiliated Hospital of \\
& Liaoning Medical Tissue \\
& Engineering Laboratory
\end{tabular}

Rabbit anti-human HIF1a monoclonal antibody, rabbit anti-human BMP2 monoclonal antibody

Secondary antibodies, $\beta$-actin

DMEM (low glucose, catalog number: 10567-014), trypsin, fetal bovine serum

A-W MGC biological scaffolds

ALP kit

Other reagents

Santa Cruz, USA

Gibco, USA

First Affiliated Hospital of Liaoning Medical Tissue Engineering Laboratory

TaKaRa, Japan

First Affiliated Hospital of Liaoning Medical Tissue Engineering Laboratory

HIF1amu hypoxia-inducible factor 1 mutation, hrGFP human renilla reniformis green fluorescent protein, BMP2 bone morphogenetic protein 2, ALP alkaline phosphatase, A-W MGC apatite-wollastonite magnetic bioactive glass-ceramic

evaluated with inverted fluorescence microscope $72 \mathrm{~h}$ after the infection.

\section{Experimental groups}

Group A: BMSCs were infected with viral solution containing Adv-BMP2-hrGFP and Adv-HIF1 $\alpha^{\mathrm{mu}}$ hrGFP.

Group B: BMSCs were infected with viral solution containing Adv-BMP2-hrGFP.

Group C: BMSCs were infected with viral solution containing Adv-HIF1 $\alpha^{\text {mu }}$-hrGFP.

Group D: BMSCs were infected with viral solution containing Adv-hrGFP.

Group E: BMSCs without infection of any viral solution.

\section{Detection of the cell ALP activity and concentration}

The supernatant in 96-well culture plates was removed $3,6,9$, and 12 days after infection; the cells were washed once with PBS. The number of cells per well was estimated and adjusted to a similar value for each well. $100 \mu \mathrm{L} 0.1 \%$ Triton X-100 was added to each well and incubated at $4{ }^{\circ} \mathrm{C}$ overnight. All procedures were carried out according to the manufacturer's instructions. The absorbance change per minute $(\Delta \mathrm{A} / \mathrm{min})$ was measured at $405 \mathrm{~nm}$ and monitored for three consecutive minutes. The ALP (alkaline phosphatase) activity $(\mathrm{U} / \mathrm{L})=\Delta \mathrm{A} / \mathrm{min} \times 2757$. 


\section{Measurement of BMP2 and HIF1a protein expressions by Western blot}

The total protein from each group was extracted using lysis buffer and the protein concentration was measured by BCA assay. Equal amounts of total protein from each group were separated by SDS-PAGE (5 and $8 \%), 60 \mathrm{~V} \times 30 \mathrm{~min}, 150 \mathrm{~V} \times 1 \mathrm{~h}$. After three washes, the proteins were transferred to PVDF membranes (100 mA $\times 30 \mathrm{~min}$ ). The membranes were incubated with primary antibodies $(1: 1500)$ at $4{ }^{\circ} \mathrm{C}$ overnight. The membranes were washed and incubated with secondary antibodies and developing solution in a dark room for $30 \mathrm{~min}$ at room temperature. The membranes were washed three times with triple-distilled water to terminate the color reaction. The target bands on the membranes were analyzed by gel imaging system with the reference OD values. The experiment was repeated three times to calculate the relative $\mathrm{OD}$ values.

\section{Production of bone-defect animal model Pre-operative treatment of biological scaffolds}

A-W MGC biological scaffolds, $1.5 \mathrm{~cm}$ length, $0.5 \mathrm{~cm}$ diameter, were sterilized, surface prepared, and dried. Seventy-two hours after viral infection, the BMSCs were suspended at a density of $2 \times 10^{7} / \mathrm{mL}$ and then transplanted into A-W MGCs, $5 \times 10^{6}$ each side. The A-W MGCs were rocking cultured at $37{ }^{\circ} \mathrm{C}, 5 \% \mathrm{CO}_{2}$ for $48 \mathrm{~h}$, and the cells were evenly and fully adhered to the internal space of the biological scaffolds.

\section{Animal model of bone defects}

New Zealand white rabbits were anesthetized and a 1.5$\mathrm{cm}$ segment from the middle section of the right radius was removed to create a bone-defect region. The pretreated A-W MGCs were anastomosed end-to-end to the stumps of the broken bones. After the surgery, the animals were given intramuscular injection of antibiotics
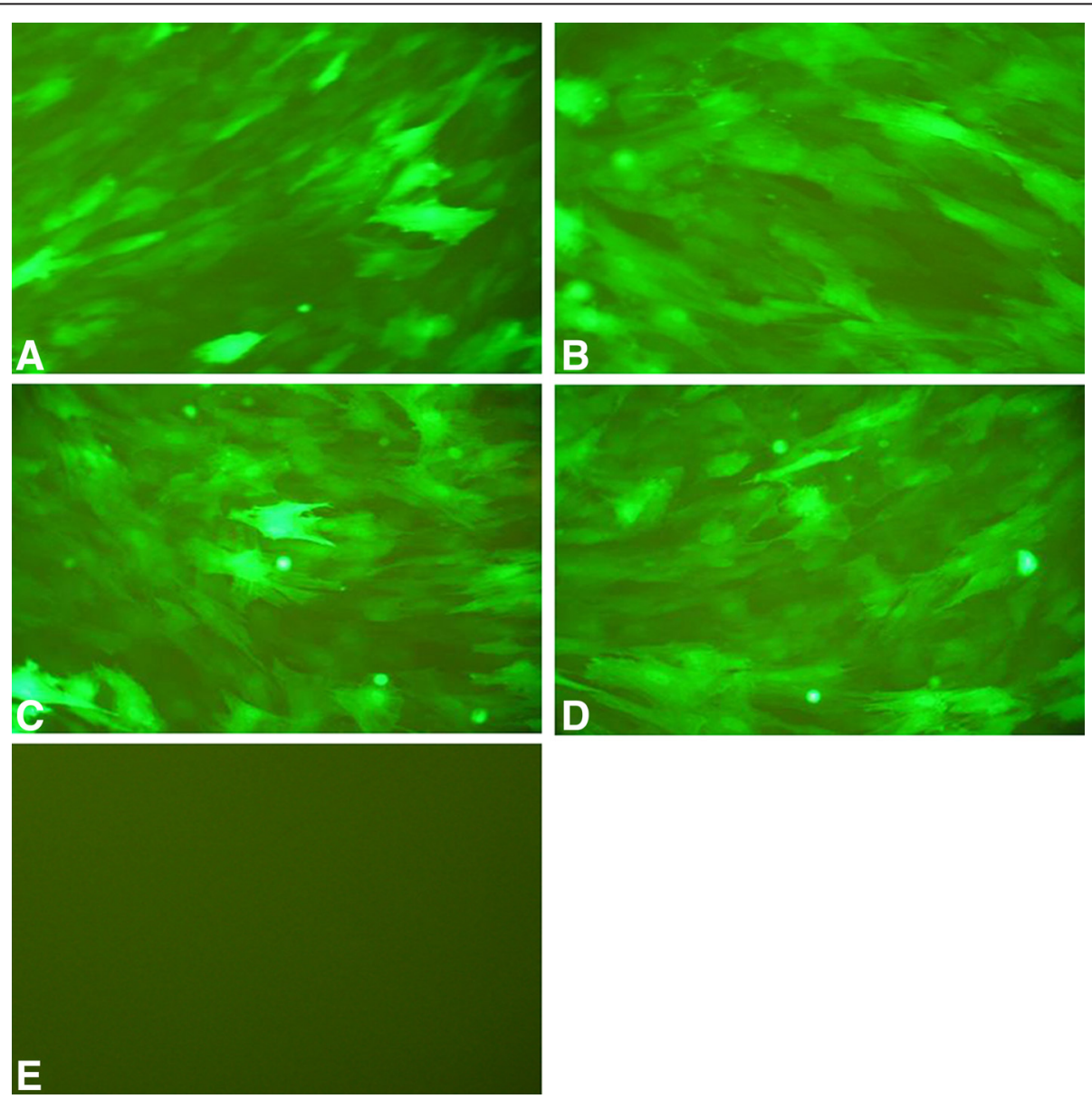

Fig. 1 The green fluorescence expressions in each group (×200). a BMSCs that were infected with Adv-BMP2-hrGFP and Adv-HIF1a ${ }^{\text {mu }}$-hrGFP. b BMSCs that were infected with Adv-BMP2-hrGFP. c BMSCs that were infected with Adv-HIF1a ${ }^{\text {mu }}$-hrGFP. d BMSCs that were infected with Adv-hrGFP. e BMSCs without infection. BMSCs: mesenchymal stem cells. BMP2: bone morphogenetic protein 2. hrGFP: human renilla reniformis green fluorescent protein. HIF1a ${ }^{\mathrm{mu}}$ : hypoxia-inducible factor 1 mutation 
for 7 days. The animals' daily activities and wound healing process were closely monitored. Animals were sacrificed 8 and 12 weeks after the surgery. All animal models were produced by the same operator.

\section{Experimental groups}

Sixty New Zealand white rabbits were randomly divided into $\mathrm{A}, \mathrm{B}, \mathrm{C}, \mathrm{D}$, and $\mathrm{E}$ groups of 12 .

Group A: A-W MGC transplanted with BMSCs that were infected with Adv-HIF $1 \alpha^{\mathrm{mu}}$-hrGFP and Adv-BMP2-hrGFP.

Group B: A-W MGC transplanted with BMSCs that were infected with Adv-BMP2-hrGFP.

Group C: autogenous bone graft group (contralateral radial transplantation).

Group D: A-W MGC transplanted with BMSCs that were infected with Adv-HIF $1 \alpha^{\mathrm{mu}}$-hrGFP.

Group E: A-W MGC scaffold alone.

\section{X-ray observation}

Eight or 12 weeks after surgery, the animals were anesthetized and the $\mathrm{x}$-ray images of the radius and ulna at the ipsilateral site of surgery were taken. Projection conditions were $40 \mathrm{kV}, 50 \mathrm{~mA}, 0.2 \mathrm{~s}$, and a projection distance of $60 \mathrm{~cm}$.

\section{Histological examination}

The animals in each group were sacrificed 12 weeks after the surgery. The radius and ulna at the bone-defect area were harvested. The degradation of the implant graft, the functional connection between the graft and the host bone, and the surrounding soft tissue response were observed. The tissues from the center of the bonedefect area were decalcified in $5 \%$ nitric acid for $72 \mathrm{~h}$, fixed in $4 \%$ formalin, paraffin-embedded, and sliced continuously with a thickness of $10 \mu \mathrm{m}$. HE staining was performed.

\section{Biomechanical measurement}

Twelve weeks after the surgery, the samples from each group were tested with a universal testing machine (AGIC, SHIMADZU) for anti-flexion strength with a span of $30 \mathrm{~mm}$ and a loading rate of $0.5 \mathrm{~mm} / \mathrm{min}$. The flexural strength of new bones at the bone-defect area in each group was compared.

\section{Statistical analysis}

SPSS 18.0 for Windows software package was used for statistical analysis. Data were expressed as mean \pm standard deviation $(x \pm s)$. One-way ANOVA was used for the comparisons among groups (normally distributed data). Non-parametric statistics (Wilcoxon rank-sum test) were used for analyzing non-normally distributed data. $p<0.05$ was considered statistically significant.

\section{Results \\ BMSCs were successfully infected with recombinant adenovirus}

The BMSCs in groups A, B, C, and D showed strong expressions of green fluorescent protein under a fluorescence microscope $72 \mathrm{~h}$ after infection. The number of BMSCs expressing green fluorescent protein was high, indicating the efficient infection rate (Fig. 1).

\section{ALP activity in each cell group}

ALP activity was detected according to the manufacturer's instructions. The absorbance change per minute $(\triangle \mathrm{A} / \mathrm{min})$ was measured at $405 \mathrm{~nm}$ and monitored for three consecutive minutes. The ALP activity $(\mathrm{U} / \mathrm{L})=$ $\Delta \mathrm{A} / \mathrm{min} \times 2757$. The ALP in group $\mathrm{A}$ was significantly higher than the $\mathrm{B}, \mathrm{C}, \mathrm{D}$, and $\mathrm{E}$ groups $(p=0.038)$. The ALP in group B was significantly higher than the C, D, and $\mathrm{E}$ groups $(p=0.041)$. There was no significant difference among $\mathrm{C}, \mathrm{D}$, and $\mathrm{E}$ groups $(p=0.926)$ (Fig. 2 and Table 2).

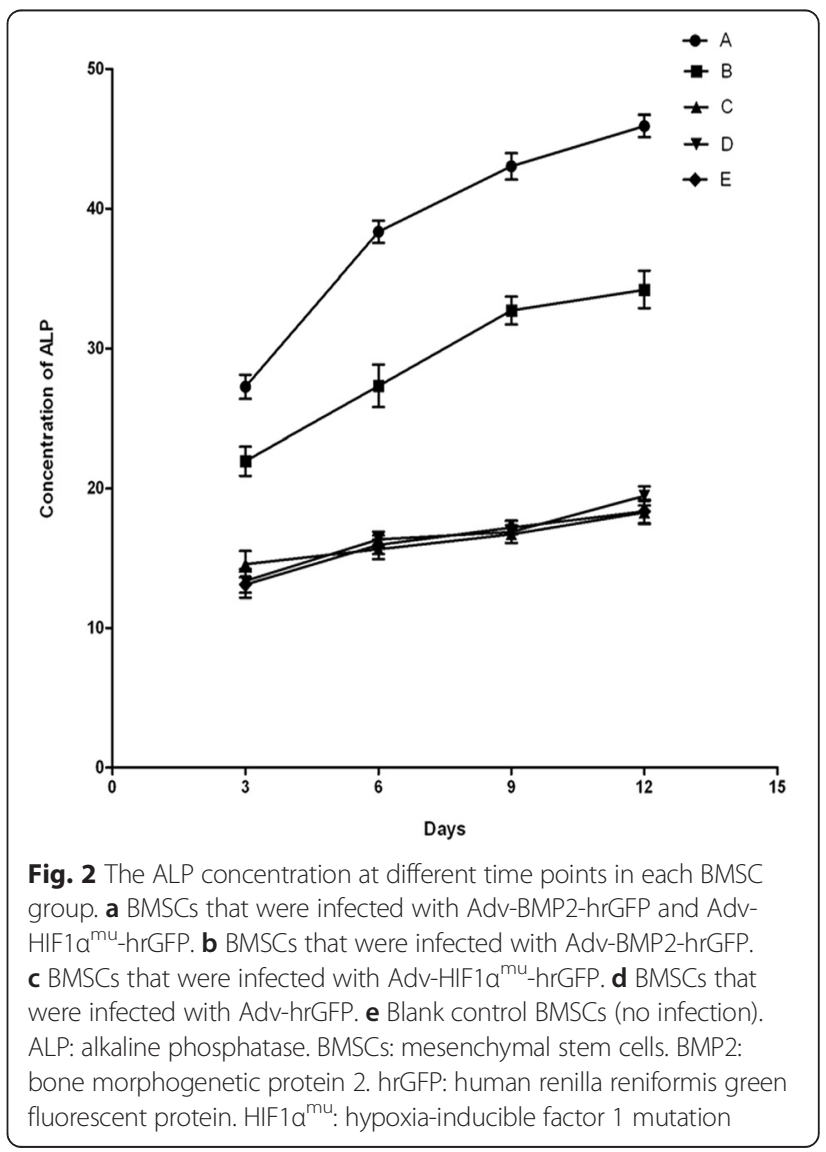


Table 2 The measurement of ALP in each BMSC group at different time points $(U / L, N=8, x \pm s)$

\begin{tabular}{lllll}
\hline Group & 3 days & 6 days & 9 days & 12 days \\
\hline A & $28.74 \pm 1.37$ & $37.89 \pm 1.73$ & $42.87 \pm 2.38$ & $45.12 \pm 2.22$ \\
B & $21.18 \pm 1.34^{*}$ & $26.91 \pm 3.62^{*}$ & $32.12 \pm 2.41^{*}$ & $33.79 \pm 2.57^{*}$ \\
C & $14.27 \pm 0.73^{* \#}$ & $15.94 \pm 1.26^{*} \#$ & $16.36 \pm 1.28^{*} \#$ & $17.15 \pm 0.74^{*} \#$ \\
D & $12.99 \pm 0.62^{* \#}$ & $16.39 \pm 0.95^{*} \#$ & $16.71 \pm 1.08^{*} \#$ & $18.01 \pm 1.24^{* \#}$ \\
E & $13.38 \pm 0.85^{*} \#$ & $16.21 \pm 1.08^{*} \#$ & $16.54 \pm 1.23^{*} \#$ & $17.08 \pm 0.91^{*} \#$
\end{tabular}

ALP alkaline phosphatase, $B M S C s$ bone marrow mesenchymal stem cells ${ }^{*}$ Compared with group $\mathrm{A}, p<0.05$; \#compared with group $\mathrm{B}, p<0.05$

\section{BMP2 and HIF1a protein expressions in each BMSCs group}

The BMP2 protein expression was significantly higher in the A and B groups, compared with groups C, D, and E $(p=0.014)$. The BMP2 protein expression was slightly higher in group A than group B; however, the difference was not significant $(p=0.069)$. The HIF1 $\alpha$ protein expression was significantly higher in the $\mathrm{A}$ and $\mathrm{C}$ groups, compared with groups $\mathrm{B}, \mathrm{D}$, and $\mathrm{E}(p=0.020)$. There was no significant difference among groups $\mathrm{B}, \mathrm{D}$, and $\mathrm{E}$, indicating that mutant HIF1 $\alpha$ can express functional proteins under normoxic conditions, whereas wild type HIF1 $\alpha$ cannot exist under normoxic conditions (Fig. 3).

\section{X-ray examination}

Eight weeks after the surgery, the bone intensity in the bone-defect area in group A (double gene infection group) and group $\mathrm{C}$ (autogenous bone graft group) significantly increased, similar to the normal bone tissue; the fracture line was barely visible, and bone defect was completely reconnected. In group B, the new bone callus increased, but the fracture line at both ends was still visible and the bone defect has not been fully reconnected. In group $\mathrm{D}$, there was a small amount of callus formation, but the bone defect has not been fully reconnected and the fracture line at both ends was clearly visible. In group $\mathrm{E}$, the implant material and the fracture line were clearly visible without signs of absorption of the implant material (Fig. 4). Twelve weeks after the surgery, the bone defect was completely repaired in group A and group $\mathrm{C}$, the bone structure was improved, and the bone density was similar to the normal bone tissue. The cortical and medullary cavity structures were visible. In group $\mathrm{B}$, the bone density in the defect area was
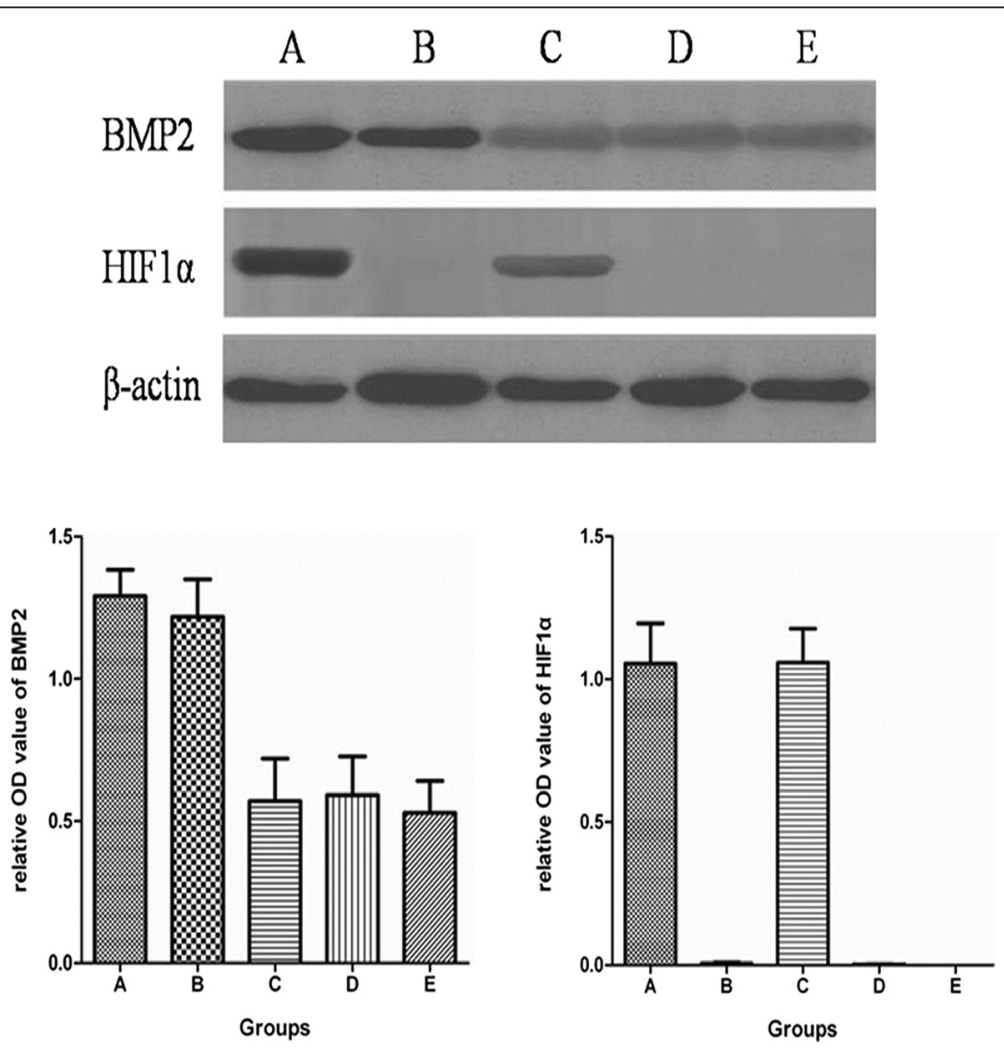

Fig. 3 The BMP2 and HIF1a protein expressions in the five BMSC groups. a BMSCs that were infected with Adv-BMP2-hrGFPand Adv-HIF1a mu hrGFP. b BMSCs that were infected with Adv-BMP2-hrGFP. c BMSCs that were infected with Adv-HIF1a ${ }^{\text {mu }}$-hrGFP. d BMSCs that were infected with Adv-hrGFP. e Blank control BMSCs (no infection). BMSCs: mesenchymal stem cells. BMP2: bone morphogenetic protein 2. hrGFP: human renilla reniformis green fluorescent protein. HIF1amu: hypoxia-inducible factor 1 mutation 


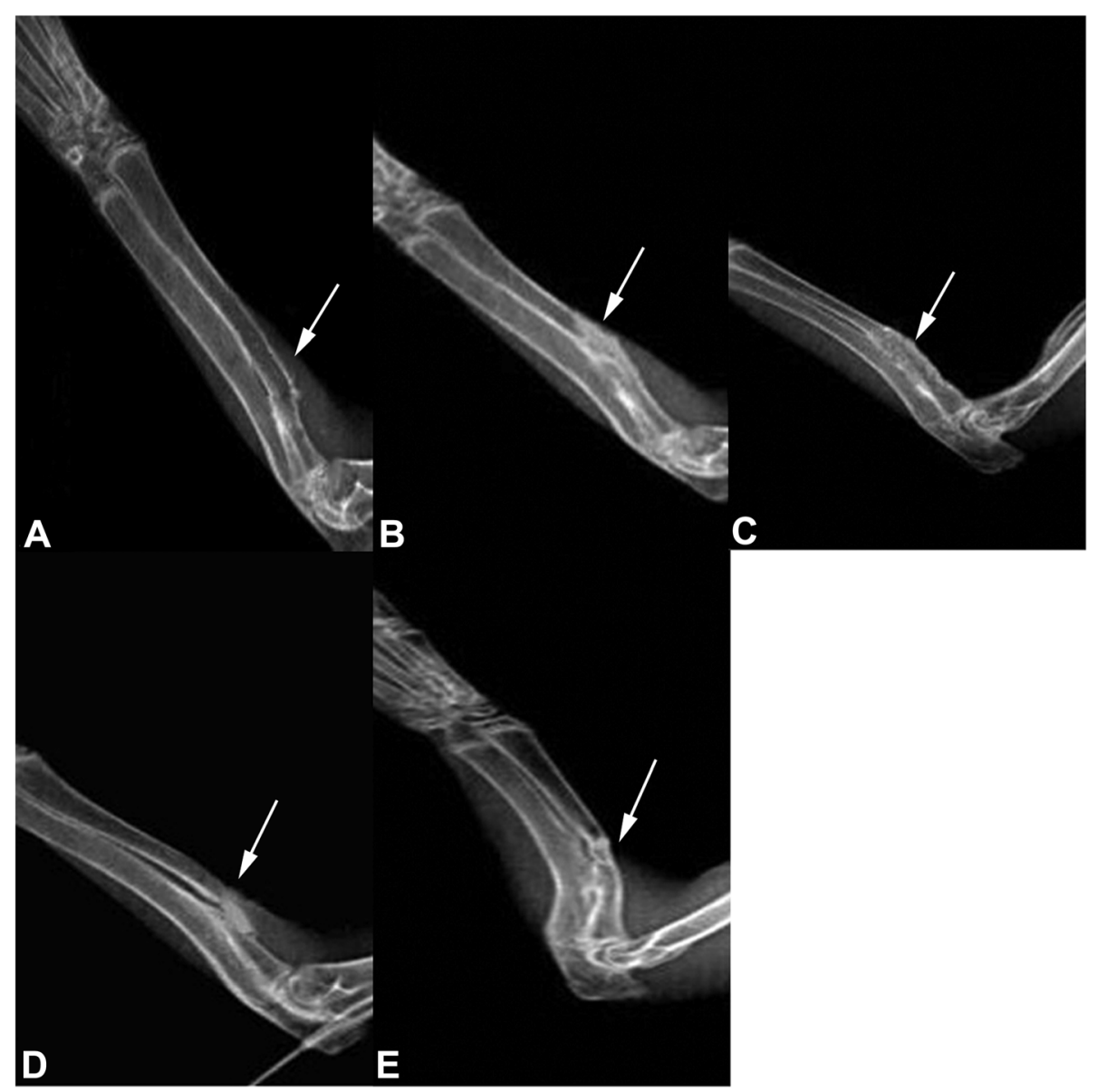

Fig. 4 X-ray showed the bone-defect areas in different groups at 8 weeks after the surgery. a A-W MGC transplanted with BMSCS that were infected with Adv-HIF1amu-hrGFP and Adv-BMP2-hrGFP. b A-W MGC transplanted with BMSCs that were infected Adv-BMP2-hrGFP. c autogenous bone graft group. d A-W MGC transplanted with BMSCs that were infected with Adv-HIF1a $a^{\text {mu}}$-hrGFP. e A-W MGC. Arrows: defect sites. MGC: magnetic bioactive glass-ceramic. BMSCs: mesenchymal stem cells. BMP2: bone morphogenetic protein 2. hrGFP: human renilla reniformis green fluorescent protein. HIF1a ${ }^{\text {mu: }}$ hypoxia-inducible factor 1 mutation

significantly increased. In group $\mathrm{D}$, the initial bonedefect repair and remodeling were slow. In group E, there was sclerosis, with no bone-defect repair or visible bone marrow cavity (Fig. 5).

\section{Histological observation}

Twelve weeks after the surgery, the A-W MGC graft was completely degraded in group A. The new bone tissues showed mature bone structure and visible trabeculae. In group $\mathrm{B}$, the bone defect was reconnected, but there was still residual carrier scaffold indicating the degradation was incomplete. In group $\mathrm{C}$, the cortex and medulla of the bone defect were completely reconnected. The bone remodeling and healing were complete. In group D, there were less trabecular bones and the carrier material was not absorbed. In group E, there was clear bone defect, poor reconnection, and visible fibrous connective tissue filling (Fig. 6).

\section{Biomechanical test (flexural strength test)}

The results of flexural strength test from each group were as follows: group A, $107.59 \pm 4.91 \mathrm{MPa}$; group $\mathrm{B}, 82.34 \pm$ $5.03 \mathrm{MPa}$; group C, $112.94 \pm 6.08 \mathrm{MPa}$; group D, $52.41 \pm$ $4.26 \mathrm{MPa}$; group $\mathrm{E}, 33.27 \pm 4.55 \mathrm{MPa}$. There was no significant difference between A and C groups $(p=0.88)$. There were significant differences between all the other two-group comparisons ( $p=0.043$ ) (Fig. 7).

\section{Discussion}

In this study, we used A-W MGC scaffold loaded with BMSCs that were infected with BMP2 and HIF1 $\alpha^{\text {mu }}$ genes for the treatment of in vivo bone defects and achieved therapeutic effects that were comparable with an autogenous bone graft. Firstly, the successful reestablishment of microcirculation system at the bone-defect site not only provides osteoblasts with nutrition but also has anti-apoptotic effects. Therefore, it will promote the stable growth of osteoblasts, accelerate bone healing 


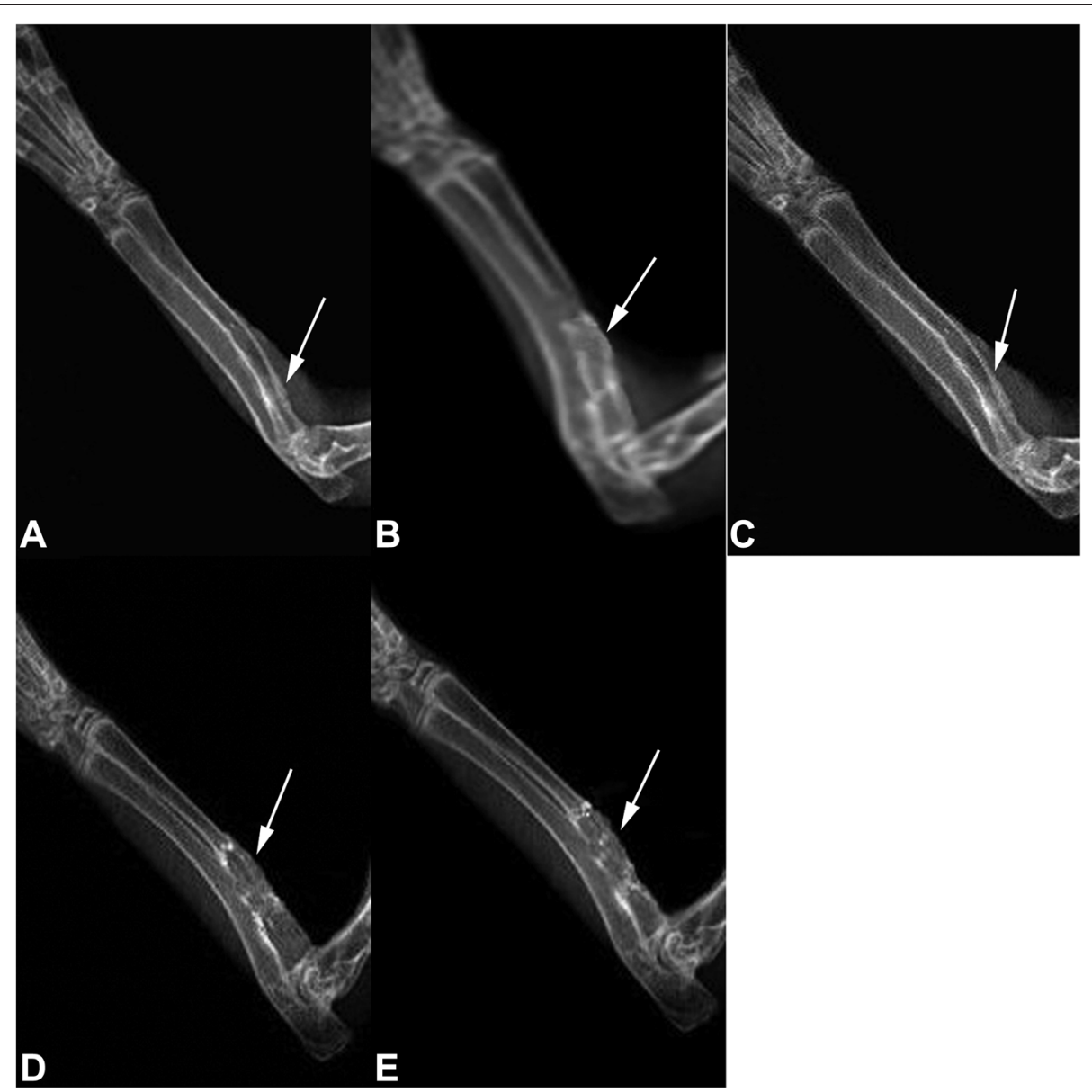

Fig. 5 X-ray showed the bone-defect areas in different groups at 12 weeks after the surgery. a A-W MGC transplanted with BMSCs that were infected with Adv-HIF1amu-hrGFP and Adv-BMP2-hrGFP. b A-W MGC transplanted with BMSCs that were infected Adv-BMP2-hrGFP. c autogenous bone graft group. d A-W MGC transplanted with BMSCs that were infected with Adv-HIF1a mu -hrGFP. e A-W MGC. Arrows: defect sites. MGC: magnetic bioactive glass-ceramic. BMSCs: mesenchymal stem cells. BMP2: bone morphogenetic protein 2. hrGFP: human renilla reniformis green fluorescent protein. $\mathrm{HIFla}^{\text {mu: }}$ : hypoxia-inducible factor 1 mutation

process, and greatly improve the survival rate of transplanted bone graft [18-20]. Secondly, a single factor may not be able to sufficiently repair the damages to the systems caused by the bone defect; in this case, it might be more sufficient to apply multiple genes to achieve individualized repair on different systems. Therefore, this experiment not only provides the foundation for the treatment of bone defects using multigene therapy, but also offers new ideas to broaden the gene therapy in other ischemic diseases.

\section{HIF1a and BMP2 as the target genes}

The key point in the gene therapy of bone defects is to introduce the osteogenic factors into the seed cells used for the tissue engineered bone graft. These seed cells can continue to proliferate in vivo and secret growth factors to promote fracture healing [21]. Among these osteogenic factors, BMP2 has the most significant effect on the bone regeneration [22-24]. Therefore, we used an adenovirus vector carrying BMP2 gene to infect BMSCs in order to achieve a sustained localized expression of bone morphogenetic protein to accelerate fracture healing.

Local application of angiogenic factors has been a part of the adjuvant treatment in bone-defect diseases and has not received much attention for the past years. However, people start to realize that it is equally important to establish a local blood circulation to prevent the death of osteoblasts after transplantation. HIF1 $\alpha$ is a transcription factor generated under hypoxic conditions and is sensitive in the detection of local tissue hypoxia. The concentration of HIF1 $\alpha$ increases with prolonged hypoxia and decreases rapidly after the improvement of hypoxia [25]. Under the temporary anoxic conditions, HIF1 $\alpha$ not only can maintain the survival of osteoblasts but also can neutralize the harmful substances produced by hypoxic cells, creating a favorable osteogenic microenvironment [25]. Therefore, HIF $1 \alpha$ is 


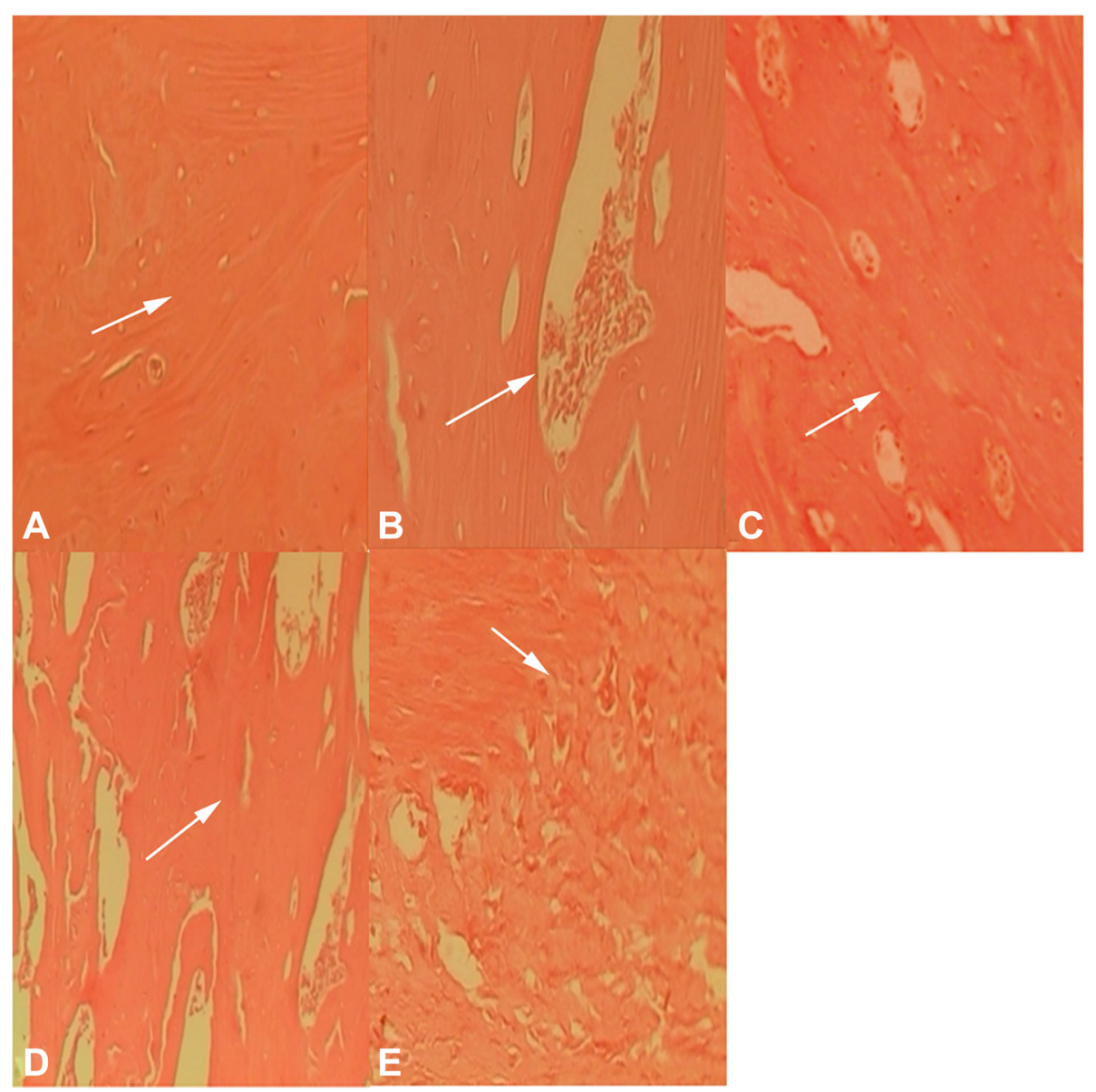

Fig. 6 HE staining images $(\times 100)$ from different groups 12 weeks after surgery. a A-W MGC transplanted with BMSCs that were infected with Adv-HIF1amu-hrGFP and Adv-BMP2-hrGFP. b A-W MGC transplanted with BMSCs that were infected Adv-BMP2-hrGFP. c Autogenous bone graft group. d A-W MGC transplanted with BMSCs that were infected with Adv-HIF1a ${ }^{\text {mu }}$-hrGFP. e A-W MGC. Arrows: cell nuclei. HE: hematoxylin and eosin. A-W MGC: apatite-wollastonite magnetic bioactive glass-ceramic. BMSCs: mesenchymal stem cells. BMP2: bone morphogenetic protein 2. hrGFP: human renilla reniformis green fluorescent protein. HIF1a ${ }^{\text {mu }}$ : hypoxia-inducible factor 1 mutation

more efficient in promoting angiogenesis than VEGF (vascular endothelial growth factor). To date, no report has been found on the effects of HIF1 $\alpha$ combined with BMP2 multigene therapy in bone-defect diseases. HIF1 $\alpha$ cannot stably express under normoxic conditions. To overcome this shortcoming, we have successfully constructed a triple mutant HIF $1 \alpha$ by point mutation of the 402,564 and 803 amino acids without affecting other functional areas. The triple point-mutant HIF1 $\alpha$ can express functional protein under normoxic conditions and play important physiological roles in angiogenesis [17].

\section{The interaction between HIF1a and BMP2}

The increase of ALP concentration is a specific marker indicating the differentiation of BMSCs into osteoblasts. In order to analyze the impact of HIF1 $\alpha^{\mathrm{mu}}$ on BMP2 expression, we measured the ALP from BMSCs that were infected with HIF1 $\alpha^{\mathrm{mu}}$ plus BMP2, the BMSCs that were infected with only BMP2, and the BMSCs without infection at 3, 6, 9, and 12 days after infection. We found that the ALP activity significantly increased in the BMSCs that were infected either with HIF $1 \alpha^{\mathrm{mu}}$ plus BMP2 or with only BMP2, compared with uninfected BMSCs. Compared with the BMSCs that were infected with only BMP2, the ALP activity in the BMSCs that were infected with HIF1 $\alpha^{\text {mu }}$ plus BMP2 was significantly higher $(p<0.05)$, suggesting that HIF1 $\alpha^{\mathrm{mu}}$ can promote BMP2 expression and increase ALP level.

\section{The advantages of A-W MGC as a scaffold}

An ideal artificial scaffold should have the following characteristics [26]: 1) has good biocompatibility (without inflammation with host tissue) and is biodegradable; 2) has excellent osteoinductive and osteoconductive effects; and 3) can provide good osteogenic environment to support the growth of seed cells. A-W MGC scaffold used in this experiment was made of bioactive ceramics and has a stronger cell adhesion by a surface treatment 


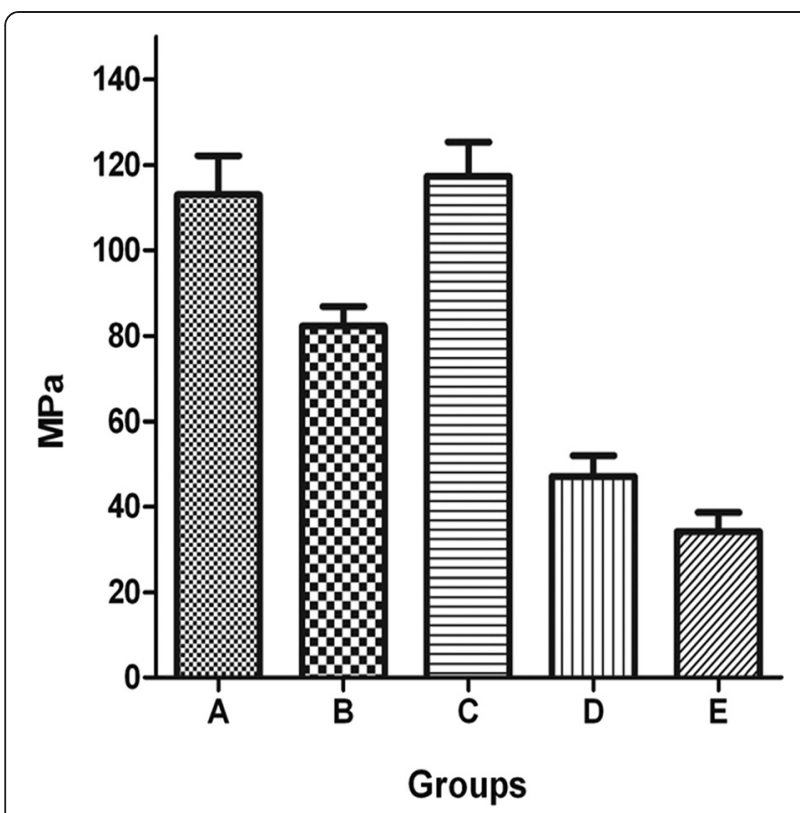

Fig. 7 The results of flexural strength test from each group. a A-W MGC transplanted with BMSCs that were infected with Adv-HIF1amuhrGFP and Adv-BMP2-hrGFP. b A-W MGC transplanted with BMSCs that were infected Adv-BMP2-hrGFP. c Autogenous bone graft group. d A-W MGC transplanted with BMSCs that were infected with Adv-HIF1amu-hrGFP. e A-W MGC. A-W MGC: apatite-wollastonite magnetic bioactive glass-ceramic. BMSCs: mesenchymal stem cells. BMP2: bone morphogenetic protein 2. hrGFP: human renilla reniformis green fluorescent protein. HIF1a ${ }^{\mathrm{mu}}$ : hypoxia-inducible factor 1 mutation

of the chitosan. A-W MGC has a large number of 2-3 $\mu \mathrm{m}$ pores, and these well-interconnected pores favor the cell adhesion and proliferation, promote angiogenesis and blood vessel network formation, and accelerate the merge of new bone and bio-material. Therefore, A-W MGC exhibits good biocompatibility, osteoconductive/ osteoinductive effects, and degradability [27], meets the requirements for bone tissue engineering material, and becomes the preferred carrier scaffold.

\section{The new tissue engineered bone graft achieved comparable therapeutic effects as the autologous bone graft}

$\mathrm{X}$-ray results indicate that the bone formation and bone remodeling capabilities in group A (A-W MGC seeded with BMSCs that were infected with HIF $1 \alpha^{\mathrm{mu}}$ plus BMP-2) were similar with group $C$ (autogenous bone graft group) $(p>0.05)$. Meanwhile, the bone formation and bone remodeling capabilities in $\mathrm{A}$ and $\mathrm{C}$ groups were superior to the other groups.

In this study, HE staining was used to observe the degradation of the implant materials, the graft-host bone interface, and the surrounding soft tissue reactions. Histological results indicate that A-W MGC in group A was fully degraded 12 weeks after surgery and the new mature bone tissues and trabecular bones were more visible. The healing of bone defect was similar compared with group $\mathrm{C}$, whereas the osteogenic effect was not very obvious in the other three groups.

Flexural strength test is the most convincing indicator for the mechanical properties of bone formation. The change in the morphology and mechanical properties of bone tissue by the external force is a reliable method for the evaluation of osteogenic effects. The results from biomechanical testing 12 weeks after surgery indicate that the engineered bone graft in group A had similar effects on the bone regeneration in the bone-defect area compared with autogenous bone graft, whereas the osteogenic effect from the other groups were much less effective.

\section{Conclusion}

In the present study, we constructed A-W MGC bone graft loaded with BMSCs that were infected with both HIF $1 \alpha^{\mathrm{mu}}$ and BMP2. The angiogenic effects of HIF $1 \alpha^{\mathrm{mu}}$ and the osteogenic effects of BMP2 interacted with each other, promoted the healing process of the bone defect, and achieved similar results as autologous bone graft. These results indicate that this A-W MGC bone graft will be an ideal alternative for autologous bone graft and solve the problems due to the insufficient number of autologous bone grafts and the complications of autologous bone transplantation. However, angiogenesis and ontogenesis are extremely complex physiological processes. The introduction of the exogenous triple pointmutant HIF1 $\alpha$ and BMP2 genes promotes the formation of blood vessel network and bone regeneration; however, the interaction of these exogenous genes with the endogenous angiogenic and osteogenic factors is still unknown. The impact of the mutation on the function of HIF $1 \alpha$ itself and BMP2, as well as the function of the three alanine mutations in the angiogenic process, needs to be further explored in our future studies.

\section{Abbreviations \\ A-W MGC: Apatite-wollastonite magnetic bioactive glass-ceramic; BMP2: Bone morphogenetic protein 2; HIF1a ${ }^{\text {mu }}$ : Hypoxia-inducible factor 1 mutation; BMSCs: Mesenchymal stem cells; MOI: Multiplicity of infection; hrGFP: Human renilla reniformis green fluorescent protein; ALP: Alkaline phosphatase; BMP: Bone morphogenetic protein; HIF1a: Hypoxia-inducible factor 1a; ODDD: Oxygen-dependent degradation domain; CDS: Coding sequence; VEGF: Vascular endothelial growth factor.}

\section{Competing interests}

The authors declare that they have no competing interests.

\section{Authors' contributions}

YG carried out the molecular genetic studies, participated in the sequence alignment, and drafted the manuscript. $C L$ participated in the design of the study and performed the statistical analysis. HW carried out the animal model studies. GF conceived of the study, participated in its design and coordination, and helped to draft the manuscript. All authors read and approved the final manuscript. 


\section{Acknowledgements}

The study was supported by the Natural Science Foundation of Liaoning Science and Technology Committee (No: 2014022010). The funding source had no role to play in the study design, the collection and interpretation of the data, writing of the report, or decision to submit this paper for publication.

\section{Author details}

'Department of Orthopedics, The Affiliated First Hospital of China Medical University, No. 155, Nanjing North Street, Heping District, 110001 Shenyang, Liaoning province, China. ${ }^{2}$ 2nd Ward of Bone and Joint, The First Affiliated Hospital of Liaoning Medical University, No.2, Wuduan, Renmin Street, 121001 Jinzhou, China. ${ }^{3}$ Biobank, The First Affiliated Hospital of Liaoning Medical University, No.2, Wuduan, Renmin Street, 121001 Jinzhou, China.

Received: 16 December 2014 Accepted: 9 May 2015

Published online: 28 May 2015

\section{References}

1. Hutchins JT, Kull Jr FC, Bynum J, Knick VC, Thurmond LM, Ray P. Improved biodistribution, tumor targeting, and reduced immunogenicity in mice with a gamma 4 variant of Campath-1H. Proc Natl Acad Sci U S A. 1995;92:11980-4.

2. Abdel-Motal UM, Friedline R, Poligone B, Pogue-Caley RR, Frelinger JA, Tisch $R$. Dendritic cell vaccination induces cross-reactive cytotoxic T lymphocytes specific for wild-type and natural variant human immunodeficiency virus type 1 epitopes in HLA-A*0201/Kb transgenic mice. Clin Immunol. 2001;101:51-8.

3. Wutzl A, Rauner M, Seemann R, Millesi W, Krepler P, Pietschmann P, et al. Bone morphogenetic proteins 2, 5, and 6 in combination stimulate osteoblasts but not osteoclasts in vitro. J Orthop Res. 2010;28:1431-9.

4. Seregin SS, Amalfitano A. Overcoming pre-existing adenovirus immunity by genetic engineering of adenovirus-based vectors. Expert Opin Biol Ther. 2009;9:1521-31.

5. Fiorenzo P, Mongiardi MP, Dimitri D, Cozzolino M, Ferri A, Montano N, et al. HIF1-positive and HIF1-negative glioblastoma cells compete in vitro but cooperate in tumor growth in vivo. Int J Oncol. 2010;36:785-91.

6. Rufaihah AJ, Haider HK, Heng BC, Ye L, Tan RS, Toh WS, et al. Therapeutic angiogenesis by transplantation of human embryonic stem cell-derived CD133+ endothelial progenitor cells for cardiac repair. Regen Med. 2010:5:231-44.

7. Giaccia A, Siim BG, Johnson RS. HIF-1 as a target for drug development. Nat Rev Drug Discov. 2003;2:803-11.

8. Khan TA, Sellke FW, Laham RJ. Gene therapy progress and prospects: therapeutic angiogenesis for limb and myocardial ischemia. Gene Ther. 2003;10:285-91.

9. Semenza GL. Evaluation of HIF-1 inhibitors as anticancer agents. Drug Discov Today. 2007;12:853-9.

10. Wang Y, Wan C, Deng L, Liu X, Cao X, Gilbert SR, et al. The hypoxiainducible factor alpha pathway couples angiogenesis to osteogenesis during skeletal development. J Clin Invest. 2007;117:1616-26.

11. Wan C, Gilbert SR, Wang Y, Cao X, Shen X, Ramaswamy G, et al. Activation of the hypoxia-inducible factor-1alpha pathway accelerates bone regeneration. Proc Natl Acad Sci U S A. 2008;105:686-91.

12. Bruick RK, McKnight SL. A conserved family of prolyl-4-hydroxylases that modify HIF. Science. 2001;294:1337-40.

13. Ceradini DJ, Yao D, Grogan RH, Callaghan MJ, Edelstein D, Brownlee M, et al. Decreasing intracellular superoxide corrects defective ischemiainduced new vessel formation in diabetic mice. J Biol Chem. 2008;283:10930-8.

14. Evans CE, Humphries J, Mattock K, Waltham M, Wadoodi A, Saha P, et al. Hypoxia and upregulation of hypoxia-inducible factor 1\{alpha\} stimulate venous thrombus recanalization. Arterioscler Thromb Vasc Biol. 2010;30:2443-51

15. Kazi AA, Molitoris KH, Koos RD. Estrogen rapidly activates the PI3KAKT pathway and hypoxia-inducible factor 1 and induces vascular endothelial growth factor A expression in luminal epithelial cells of the rat uterus. Biol Reprod. 2009;81:378-87.

16. Lando D, Peet DJ, Whelan DA, Gorman JJ, Whitelaw ML. Asparagine hydroxylation of the HIF transactivation domain a hypoxic switch. Science. 2002;295:858-61.
17. Li C, Liu D, Zhang Z, Wang G, Xu N. Triple point-mutants of hypoxiainducible factor-1alpha accelerate in vivo angiogenesis in bone defect regions. Cell Biochem Biophys. 2013;67:557-66.

18. Tran ED, Schmid-Schonbein GW. An in-vivo analysis of capillary stasis and endothelial apoptosis in a model of hypertension. Microcirculation. 2007; 14:793-804

19. Xiao YF, Wu DD, Liu SX, Chen X, Ren LF. Effect of arsenic trioxide on vascular endothelial cell proliferation and expression of vascular endothelial growth factor receptors Flt-1 and KDR in gastric cancer in nude mice. World J Gastroenterol. 2007;13:6498-505.

20. Morrison A, Brady J. Mandibular reconstruction using nonvascularized autogenous bone grafting. Curr Opin Otolaryngol Head Neck Surg. 2010;18:227-31.

21. Kim I, Kim HJ, Kim HM. Array of amorphous calcium phosphate particles improves cellular activity on a hydrophobic surface. J Biomed Mater Res B Appl Biomater. 2010;93:113-21.

22. Hsu WK, Wang JC, Liu NQ, Krenek L, Zuk PA, Hedrick MH, et al. Stem cells from human fat as cellular delivery vehicles in an athymic rat posterolateral spine fusion model. J Bone Joint Surg Am. 2008;90:1043-52.

23. Liu D, Hu L, Zhang Z, Li QY, Wang G. Construction of human BMP2-IRESHIF1alphamu adenovirus expression vector and its expression in mesenchymal stem cells. Mol Med Rep. 2013;7:659-63.

24. Fang J, Yang Z, Tan S, Tayag C, Nimni ME, Urata M, et al. Injectable gel graft for bone defect repair. Regen Med. 2014;9:41-51.

25. Simon F, Bockhorn M, Praha C, Baba HA, Broelsch CE, Frilling A, et al. Deregulation of HIF1-alpha and hypoxia-regulated pathways in hepatocellular carcinoma and corresponding non-malignant liver tissue-influence of a modulated host stroma on the prognosis of HCC. Langenbeck's Arch Surg / Deutsche Gesellschaft fur Chirurgie. 2010;395:395-405.

26. Teramoto H, Kawai A, Sugihara S, Yoshida A, Inoue H. Resorption of apatitewollastonite containing glass-ceramic and beta-tricalcium phosphate in vivo. Acta Med Okayama. 2005;59:201-7.

27. Udoh K, Munar ML, Maruta M, Matsuya S, Ishikawa K. Effects of sintering temperature on physical and compositional properties of alpha-tricalcium phosphate foam. Dent Mater J. 2010;29:154-9.

\section{Submit your next manuscript to BioMed Central and take full advantage of:}

- Convenient online submission

- Thorough peer review

- No space constraints or color figure charges

- Immediate publication on acceptance

- Inclusion in PubMed, CAS, Scopus and Google Scholar

- Research which is freely available for redistribution 